\title{
Hand sanitizers may increase norovirus risk
}

A lcohol-based hand sanitizers may not be the panacea for hand hygiene they were once supposed, as mounting research indicates they may not be effective substitutes for soap and water, and in some cases may actually increase the risk for outbreaks of highly contagious viruses in health care settings.

Public health experts, however, say more rigorous investigations will be necessary to trump the convenience of using hand sanitizers, among other benefits, or to substantially alter existing recommendations that strongly encourage their use by health care professionals.

It's widely recognized that improper use of antibiotics contributes greatly to the development and spread of super bugs in health care settings, but the link between hand sanitizers and bacterial resistance is less clear.

A survey of 161 long-term care facilities in the United States presented at an American College of Preventative Medicine meeting in February revealed an association between the preferential use of alcohol-based hand sanitizers for routine hand hygiene with an increased risk for outbreaks of norovirus, the highly infectious virus that causes most cases of acute gastroenteritis.

Staff in facilities that experienced norovirus outbreaks were six times more likely to use hand sanitizers equally or more than soap and water for routine hand hygiene, according to the study (Am J Infect Control 2011;39:296-301). Of 45 facilities that reported preferential use of alcohol-based hand sanitizers, $53 \%$ experienced a confirmed outbreak of norovirus, compared with $18 \%$ of 17 facilities that used hand sanitizers less often than soap and water.

Moreover, three facilities with multiple norovirus outbreaks reported that staff were more likely or much more likely to use hand sanitizers than soap and water, both routinely and during an outbreak.

While these findings indicate that alcohol-based hand sanitizers might be

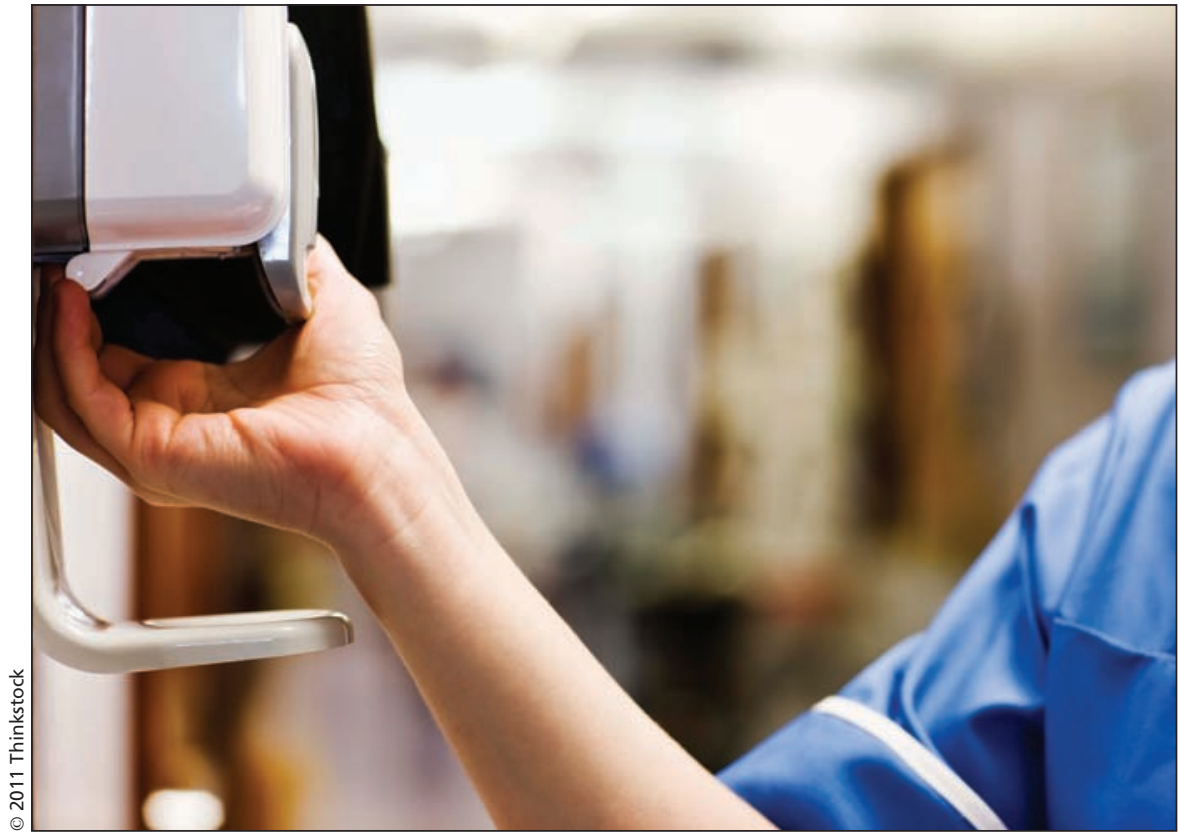

Of the $\mathbf{4 5}$ facilities that reported preferential use of alcohol-based hand sanitizers in a recent survery, $53 \%$ experienced a confirmed outbreak of norovirus, compared with $18 \%$ of the 17 facilities that used hand sanitizers less often than soap and water.

"suboptimal in controlling the spread of noroviruses," the retrospective design of the survey precludes any causal link between hand sanitizer use and norovirus outbreaks, says author Dr. David Blaney of the Epidemic Intelligence Service at the US Centers for Disease Control and Prevention (CDC).

Care homes that reported preferential use of hand sanitizers might simply have more robust infection control programs and therefore might be more likely to recognize and report norovirus outbreaks, he explains. "That being said, this study shows there's a need to look at more organism-specific outbreaks prospectively."

If the findings are confirmed in prospective studies, Blaney says changes to recommended policies regarding routine hand hygiene practices should be considered to place increased emphasis on hand washing using soap and water, as well as the use of disposable gloves during outbreaks.

Other studies have demonstrated that alcohol-based hand sanitizers are often ineffective against nonenveloped viruses, including norovirus, suggesting their use may not be appropriate in settings that frequently experience outbreaks, such as long-term care facilities (Appl Environ Microbiol 2010; 76:394399 and Appl Environ Microbiol 2008;74:5047-52).

Two studies looking at the effectiveness of alcohol-based hand sanitizers against Clostridium difficile have also indicated that overreliance on the sanitizers may leave viable spoors on the hands of health care workers (Infect Control Hosp Epidemiol 2010; 31:56570 and Infect Control Hosp Epidemiol 2009; 30:239-44).

The CDC has since released updated guidelines for the prevention and control of norovirus that recommend against using hand sanitizer as a substitute for soap and water hand washing (www.cdc.gov/mmwr/preview/mmwrht $\mathrm{ml} / \mathrm{rr6003a1.htm)}$.

But while "the efficacy of alcoholbased and other hand sanitizers against norovirus remains controversial," the 
CDC's Updated Norovirus Outbreak Management and Disease Prevention Guidelines suggest that the evidence is by no means conclusive.

"The findings of these studies also have to be taken in context of the benefits afforded by alcohol-based hand sanitizers and the uncertainty in their designs," explains Aron Hall, an epidemiologist with the CDC's division of viral diseases. "We can, however, strongly recommend more prospective studies to evaluate the issue further."

The use of alcohol-based hand sani- tizers has greatly increased adherence to hand hygiene in health care settings, says Kate Ellingson, another epidemiologist with the CDC's division of health care quality promotion. "Hand sanitizer is promoted so widely because it's efficient, accessible and takes relatively very little time to use. There's potentially a large tradeoff in switching back to soap and water in the health care setting, where workers have to wash their hand many, many times per shift, or even hour, depending on what they're doing."
Releasing stronger recommendations against sanitizers could result in a significant backlash from health care professionals and affect gains in hand hygiene adherence, Ellingson adds. "If some kind of association was found through more rigorous trials, we would want to better understand the particular indications when the risk was highest so as not to totally throw the baby out with the bathwater." - Lauren Vogel, CMAJ

CMAJ 2011. DOI:10.1503/cmaj.109-3922 